\title{
A UNIVERSAL VECTOR CARDIOGRAPH
}

BY

\author{
A. G. TH. BECKING, H. C. BURGER, AND J. B. VAN MILAAN
}

From the Physisch Laboratorium der Rijks Universiteit te Utrecht, Holland

Received April 4, 1950

The electric action of the heart can be represented by a vector (heart-vector), varying during the heart beat in magnitude and direction. If this vector is drawn from a fixed zero point, the end point describes a three-dimensional curve-the vector cardiogram. Several investigators have constructed a vector cardiograph, i.e. an instrument designed to visualize or photograph one or more projections of this curve (Cantonnet and Milovanovitch, 1947; Duchosal and Sulzer, 1949; Hollmann and Hollmann, 1938; Schellong, 1939; Vastesaeger and Rochet, 1944; Wilson and Johnston, 1938). As these must be derived from electrocardiographic leads, the method depends on the relation between heart-vector and leads. Some investigators have used the well-known triangle rule of Einthoven; others have not used extremity leads according to Einthoven, but have placed the electrodes in such a way, that the lines connecting them are mutually perpendicular. All these methods are founded on arbitrary suppositions. It is, however, possible to generalize these methods and to use a linear relation between heart-vector and leads (Burger and van Milaan, 1946, 1947, 1948). Using three leads, this gives three linear equations for the components $X, Y, Z$ of the heart-vector, from which the latter can be solved:

$$
\begin{aligned}
& X=\alpha_{1}(\text { lead })_{1}+\alpha_{2}(\text { lead })_{2}+\alpha_{3}(\text { lead })_{3} \\
& Y=\beta_{1}(\text { lead })_{1}+\beta_{2}(\text { lead })_{2}+\beta_{3}(\text { lead })_{3} \\
& Z=\gamma_{1}(\text { lead })_{1}+\gamma_{2}(\text { lead })_{2}+\gamma_{3}(\text { lead })_{3}
\end{aligned}
$$

We shall not consider here how it is possible to obtain information about the nine coefficients $\alpha_{1}, \ldots \gamma_{3}$. We shall consider them as numerically known constants in this paper.

Principle of the instrument. It is possible to obtain tensions, proportional to the components $X, Y, Z$ of the heart-vector, as given by equations (1). Indeed, in these equations two mathematical operations are indicated, namely, a multiplication of the three leads by the coefficients $\alpha_{1}, \ldots \gamma_{3}$, and an addition of the products. Now multiplication of a lead (potential difference) by a factor is easily performed by amplification with a radio-valve if the factor is greater than one and by a resistance (a so-called potentiometer) if it is smaller than one. There remains the additions of the three terms in each of the equations (1).

Addition of three potential differences can be obtained by the arrangement, represented in Fig. 1. Let $A, B, C$ be the three points, the potential differences of which with an arbitrary point are to be added. Then $A, B, C$ are connected to a common point $P$ by three equal resistances $R$. If these resistances are large enough -with respect to the internal resistances in the system $A B C$, the potential difference of $P$ with the arbitrary point is the average of the three potential differences of $A, B, C$, with this point. This can easily be proved with the laws of Ohm and Kirchhoff.* Then the average can be transformed into the required sum by multiplication by three. The sensitivity is therefore reduced to one-third of the original value or the amplification has to be trebled.

* Compare the well-known Wilson central terminal. 
This method allows only the addition of potential differences of the same sign. As in general the signs are different, the method has to be extended. To this end a so-called push-pull amplifier is used, that is a combination of two radio-valves and two resistances arranged in parallel as a Wheatstone bridge. A voltage on the grids of each of these valves gives deflections of an opposite sign. Now the sum of the positive voltages is applied to one grid, the sum of the negative ones to the other. In Fig. $1, P$ is the grid, and the points $A, B, C$ are to be connected to the positive voltages or to zero potential.

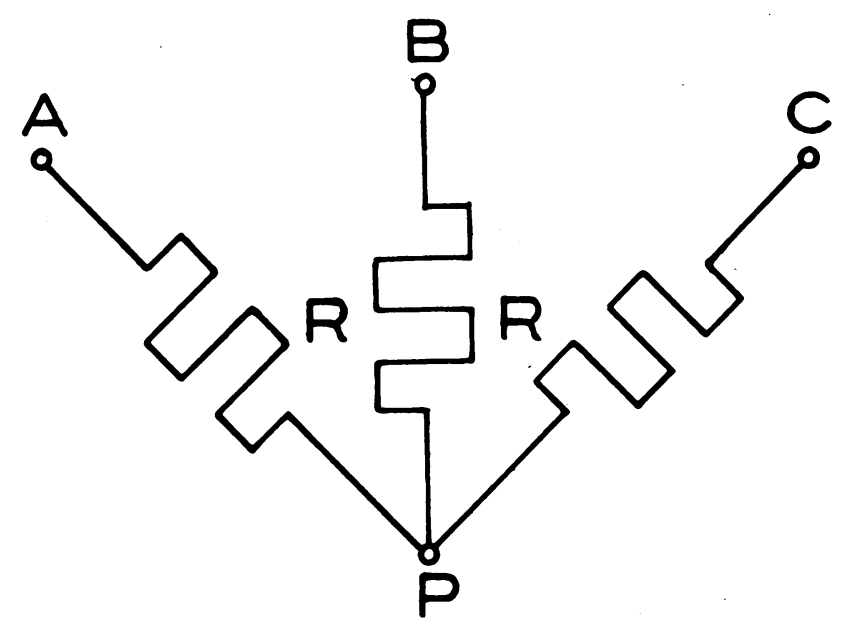

FIG. 1.-If the three resistances $R$ are equal, the potential of $P$ is the average of the potentials of $A, B$, and $C$.

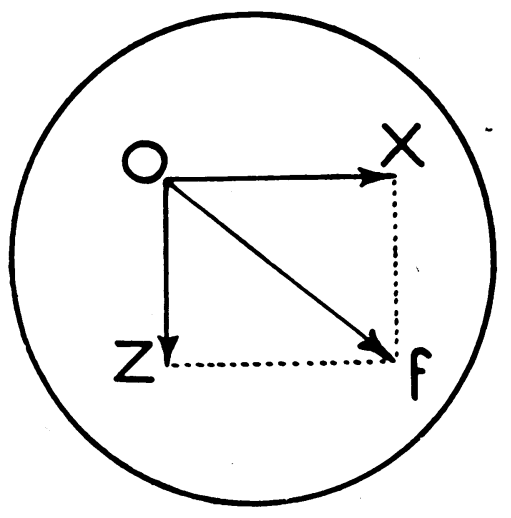

FIG. 2.- $O X$ and $O Y$ are deflections of the light spot on the screen of a cathode-ray tube, proportional to horizontal and vertical component of the heart-vector. The spot is deflected to $f$.

Design of the instrument. The instrument consists of two separate parts, a pre-amplifier and the instrument proper with the cathode-ray tubes. The first part contains four two-stage, resistancecapacity coupled amplifiers. The input resistance is $1 \mathrm{M} \Omega$, high compared with the outer resistance, even with small electrodes. Behind the first valve of each amplifier the voltage can be reduced in five steps to 2 per cent of the original one. Behind the second valve a cathode follower reduces the output resistance, in order to make it small compared with the input resistance of the following stage. This part of the instrument is fed by accumulators and anode batteries, although the latter ones could easily be replaced by mains units.

On the front plate of the second part of the instrument, sixteen knobs serve to adjust the values of the coefficients. Under each knob is a small one by which to choose the sign of the coefficient. In this way it is possible to obtain four voltages, each being a linear function of the four entrance voltages, combined with the required coefficients. As a rule only three entrance voltages are used.

The frontal projection of the vector cardiogram is obtained in the following manner. A voltage proportional to the horizontal component X (Fig. 2) of the heart-vector (pointing from the right to the left) is applied to the vertical condensor plates, which give the light spot on the screen of the cathode-ray tube a deflection to the right. The component $Z$ simultaneously gives a deflection downwards so that the spot is displaced to $f$. The vector $O f$, pointing from the zero point $O$ to the actual position $f$ of the spot represents the frontal projection of the heart-vector. During the heart beat the light spot describes a curve, which is the frontal projection of the three-dimensional vector cardiogram.

The instrument is provided with two cathode-ray tubes. By properly adjusting the knobs the second tube can give another projection of the vector cardiogram. We use as a rule the horizontal one, giving a view of the vector cardiogram seen from the head end.

The instrument is calibrated with an alternating voltage of $1 \mathrm{mV}$. With a switch this voltage 
is connected successively with the three entrance electrodes. On each screen there appears a straight, inclined line, which, by turning the knobs is given the right components in a horizontal and a vertical direction. As the coefficients can be given in an absolute measure, the same is true for the vector cardiogram. There would be no sense in mentioning the deflection on the screen corresponding to $1 \mathrm{mV}$, as this depends on the component and on the position of the entrance electrode. It is, however, possible to give the deflection corresponding to the unit of dipole momentum, the Volt $\mathrm{cm}^{2}$, which we choose as a rule equal to a few centimetres.

The time is visualized by making the electron beam intermittent, so that a dotted line appears on the screen. The time interval can be adjusted between 1 and $50 \mathrm{msec}$. $(\sigma)$. The intensity of each dot increases rapidly and falls off slowly, giving the direction in which the spot is moving.

To protect the screen during the stationary position or slow motion of the light spot, the intensity can be regulated automatically. The greater the velocity, the greater is the intensity of the electron beam. In this way the contrast in the photographic image can be reduced to such an extent, that the intensity of the slower P and T is not too high, if that of the quick QRS loop is sufficiently increased.

With two lenses slightly reduced images of both screens are projected on photographic paper. As a rule some photographs are made with and without intermittence of the beam. If the dipole momentum is small the photographs are made with a higher amplification.

Results. In the above-mentioned form of two projections the result is not quite clear at once. We, therefore, make a loop of copper wire, the projections of which agree with the photographic images of the horizontal and vertical projections.

As an example Fig. 3 shows the photographic image of frontal and top view of a loop of which Fig. 4 gives the stereoscopic image of its wire model.

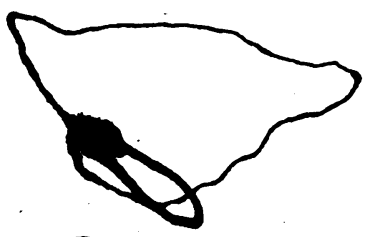

a

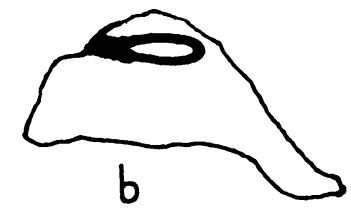

FIG. 3.-Photographic image of horizontal (a, left) and frontal (b, right) projection of a vector-cardiogram.
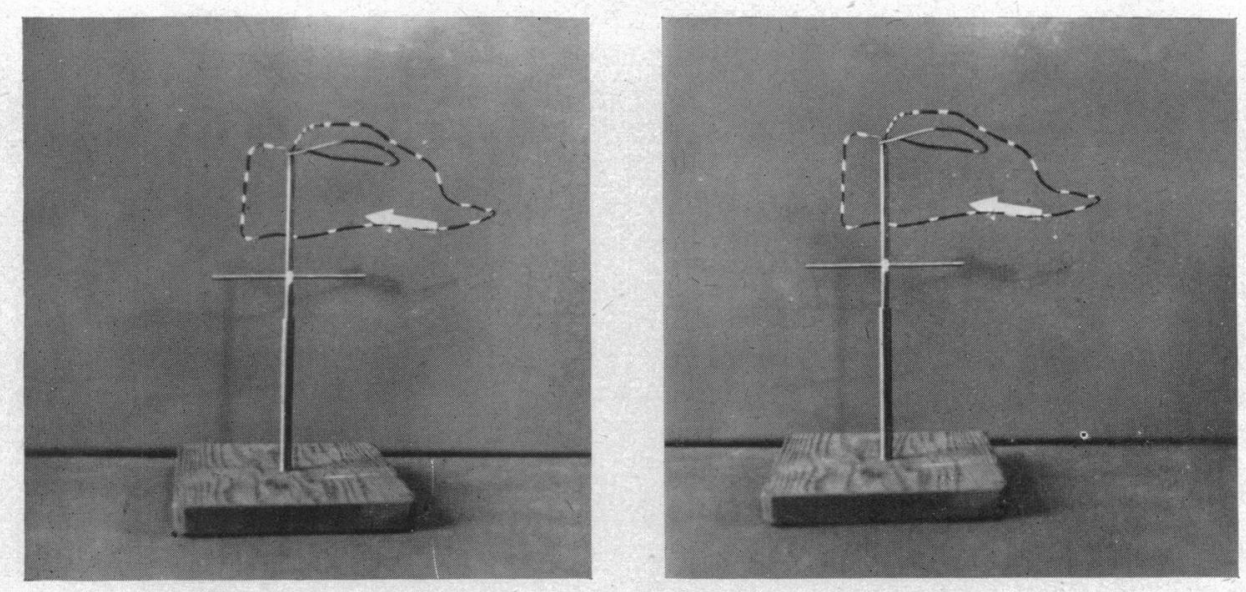

FIG. 4.-Stereoscopic image of a wire model of the vector cardiogram of Fig. 3. 
The instrument is designed for universal use. We have made vector cardiograms with different positions of the electrodes, using for each set of electrodes the coefficients as determined with the aid of a phantom (Burger and van Milaan, 1947). But if one should want to use other coefficients, they can easily be altered by turning the knobs. The well-known Einthoven frontal view of the vector cardiograms can be obtained by choosing the proper coefficients. Also vector cardiograms according to the suppositions of Duchosal and Sulzer (1949) can be and have indeed been made.

The writers are indebted to Mr. G. Casteleyn and Mr. F. R. Diemont, for the trouble and the time spent in working out the electronic circuits and in constructing the instrument.

\section{REFERENCES}

Burger, H. C., and van Milaan, J. B. (1946). Brit. Heart J., 8, 157. (1947). Ibid., 9, 154. (1948). Ibid., 10, 229 .

Cantonnet, P., et Milovanovich, J. B. (1947). Compt. Rend. Soc. Biol., 141, 613.

Conway, J. F., Cronvich, J. A., and Burch, G. E. (1949). Amer. Heart J., 38, 537.

Duchosal, P. W., et Sulzer, R. (1949). La Vector-cardiographie. Karger, Bâle.

Hollmann, H. E., und Hollmann, W. (1938). Ztsch. Klin. Med., 134, 732.

Schellong, F. (1939). Grundzüge einer klinischen Vektordiagraphie des Herzen. Springer, Berlin.

Vastesaeger, M. M., et Rochet, J. (1944). Trav. Lab. Inst. Solvay Physiol., 29, fasc. 4.

Wilson, F. N., and Johnston, F. D. (1938). Amer. Heart J., 16, 14. 